\title{
ON A THEOREM OF PREISSMANN
}

\author{
WILLIAM P. BYERS
}

In this note we strengthen a theorem of Preissman [3] which states that the only abelian subgroups of the fundamental group of a compact manifold of negative curvature are infinite cyclic. Our result is

Theorem. Let $M$ be a compact Riemannian manifold with all sectional curvatures less than zero. If $H$ is a solvable subgroup of $\pi_{1}(M)$ then $H$ must be infinite cyclic. In particular $\pi_{1}(M)$ has no solvable subgroup of finite index.

Let $\pi: \bar{M} \rightarrow M$ be a universal covering of $M$ with the induced metric on $\bar{M}$. We identify the fundamental group $\pi_{1}(M)$ with the group of all covering transformations of this covering. We need the following (well-known) lemmas:

Lemma 1. Let $\alpha: \bar{M} \rightarrow \bar{M}$ be any covering transformation. Then $\alpha$ maps some geodesic of $\bar{M}$ into itself.

Proof. Let $x_{0} \in M$ and $\bar{x}_{0} \in \bar{M}$ such that $\pi\left(\bar{x}_{0}\right)=x_{0}$. Let $\bar{C}$ be a path in $\bar{M}$ joining $\bar{x}_{0}$ to $\alpha\left(\bar{x}_{0}\right)$ and let $C$ be its projection into $M$. According to Bishop and Crittenden [1, p. 293] there is a closed geodesic in every free homotopy class of loops on $M$. Let $C_{1}$ be a closed geodesic which is free homotopic to $C$ and let $F:[0,1] \times[0,1] \rightarrow M$ be the free homotopy joining $C$ to $C_{1}$, i.e. $F(x, 0)=C(x), F(x, 1)=C_{1}(x), F(0, t)$ $=F(1, t)$. Let $G(t)=F(0, t)$ and let $\bar{G}$ be the unique lift of this path to $\bar{M}$ such that $\bar{G}(0)=\bar{x}_{0}$. Let $\bar{G}(1)=\bar{y}_{0}$. It follows from elementary properties of covering spaces that the loop $C_{1}$ and the base point $\bar{y}_{0}$ induce the transformation $\alpha$. However, the former transformation obviously preserves the geodesic in $\bar{M}$ which passes through $\bar{y}_{0}$ and covers $C_{1}$. Q.E.D.

LEMMA 2. Each covering transformation preserves at most one geodesic of $\bar{M}$.

Proof. Let $C_{1}$ and $C_{2}$ be two geodesics preserved by $\alpha \in \pi_{1}(M)$. Then $C_{1} \cap C_{2}=\varnothing$ since $\alpha$ would fix any point of intersection. If $t \in R$ is a parametrization by arc length along $C_{1}$ then (E. Cartan [2]) the function $f(t)$ which measures the distance to $C_{2}$ achieves no local maxima. Thus it must be either increasing, decreasing, or decreasing to a minimum and then increasing. However, the distance of $x \in C_{1}$

Received by the editors June 13, 1969. 
to $C_{2}$ equals the distance from $\alpha^{n}(x)$ to $C_{2}$ since $\alpha$ is an isometry. Thus the function $f$ reduces to a constant function which is impossible on a manifold of strictly negative curvature. Q.E.D.

PROOF OF THE THEOREM. Let $H=H_{0} \supset H_{1} \supset H_{2} \supset \cdots \supset H_{k-2}$ $\supset H_{k-1} \supset H_{k}=\{1\}$ be the derived series for $H$. Let $\alpha, \beta \in H_{k-1}$ which is abelian and suppose $\alpha$ preserves the geodesic $C$. Then $\alpha(\beta(C))$ $=\beta(\alpha(C))=\beta(C)$. From Lemma $2, \beta(C)=C$. This implies that $\alpha$ and $\beta$ are generated by the same closed geodesic on $M$. Suppose $\gamma$ is the transformation which corresponds to going around this closed geodesic exactly once, with $\gamma(C)=C$. Then we have shown that $H_{k-1}$ $\subseteq\left\{\gamma^{n}\right\}_{n \in Z}$. Thus $H_{k-2}$ is infinite cyclic if $\left\{\gamma^{n}\right\}_{n \in Z}$ is. That $\left\{\gamma^{n}\right\}_{n \in Z}$ is infinite cyclic follows from the fact that $\bar{M}$ is diffeomorphic to Euclidean space and a result of P. A. Smith [4] stating that $Z_{p}$ cannot act freely on $R^{n}$.

Let $\delta \in H_{k-2}$. Then $\delta^{-1} \alpha^{-1} \delta \alpha=\gamma^{n}$ for some $n \in Z$. Thus $\delta^{-1} \alpha^{-1} \delta \alpha(C)$ $=\gamma^{n}(C)=C$, i.e. $\alpha^{-1} \delta(C)=\delta(C)$. Therefore $\alpha$ preserves $\delta(C)$ and $\delta(C)=C$ as above. Again this implies that $\delta=\gamma^{m}$ for some $m \in Z$ which shows that $H_{k-z} \subseteq\left\{\gamma^{n}\right\}$. Proceeding in this way we eventually show that $H \subseteq\left\{\gamma^{n}\right\}_{n \in Z}$ and therefore $H$ is infinite cyclic.

To prove the final statement, let $\left\{\gamma^{n}\right\}_{n \in Z}$ be the solvable subgroup of finite index which we now know to be infinite cyclic. Suppose $\alpha$ is some transformation not in that subgroup. Then $\alpha^{n}=\gamma^{m}$ for some $n, m \in Z$ since the subgroup has finite index. Suppose $\gamma(C)=C$. Then $\alpha^{n}(C)=C$. However, since $\alpha$ preserves a unique geodesic we must have $\alpha(C)=C$. As above this implies that $\pi_{1}(M)$ is infinite cyclic. However, this is impossible for compact $M[3$, p. 206].

\section{REFERENCES}

1. R. L. Bishop and R. J. Crittenden, Geometry of manifolds, Pure and Appl. Math., vol. 15, Academic Press, New York, 1964. MR 29 \#6401.

2. E. Cartan, Leçons sur la géométrie des espaces de Riemann, 2nd ed., GauthierVillars, Paris, 1951. MR 13, 491.

3. A. Preissmann, Quelques propriêtés globales des espaces de Riemann, Comment. Math. Helv. 15 (1943), 175-216. MR 6, 20.

4. P. A. Smith, "Fixed points of periodic transformations," Appendix B in Lefschetz, Algebraic topology, Amer. Math. Soc. Colloq. Publ., vol. 27, Amer. Math. Soc., Providence, R. I., 1942. MR 4, 84.

University of California, Berkeley 